Published in final edited form as:

Curr Opin Virol. 2015 April ; 11: 7-13. doi:10.1016/j.coviro.2015.01.003.

\title{
Reassessing Immune Control of Hepatitis A Virus
}

\author{
Christopher M. Walker ${ }^{1,2}$, Zongdi Feng ${ }^{1,2}$, and Stanley M. Lemon ${ }^{3,4,5}$ \\ ${ }^{1}$ Center for Vaccines and Immunity, Nationwide Children's Hospital, Columbus Ohio, 43205 Ohio \\ ${ }^{2}$ College of Medicine, The Ohio State University, Columbus Ohio, 43205 Ohio \\ ${ }^{3}$ Department of Medicine, Division of Infectious Diseases, The University of North Carolina at \\ Chapel Hill, Chapel Hill, North Carolina, USA \\ ${ }^{4}$ Lineberger Comprehensive Cancer Center, The University of North Carolina at Chapel Hill, \\ Chapel Hill, North Carolina, USA \\ ${ }^{5}$ Department of Microbiology and Immunology, The University of North Carolina at Chapel Hill, \\ Chapel Hill, North Carolina, USA
}

\begin{abstract}
There is renewed interest in hepatitis A virus (HAV) pathogenesis and immunity after 2-3 decades of limited progress. From a public health perspective, the average age at infection has increased in developing countries, resulting in more severe hepatitis that is poorly understood mechanistically. More fundamentally, there is interest in comparing immunity to HAV and hepatitis $\mathrm{C}$ virus (HCV): small, positive-strand RNA viruses with very different infection outcomes. Here, we review evidence that circulating HAV virions are cloaked in membranes, with consequences for induction of innate immunity and antibody-mediated neutralization. We also consider the contribution of CD4+ helper versus CD8+ cytotoxic T cells to antiviral immunity and liver injury, and present a model of non-cytotoxic immune control of HAV infection.
\end{abstract}

\section{Introduction}

Hepatitis A virus (HAV) is a positive-strand RNA virus classified in the family Picornaviridae. A prominent cause of fecal-orally transmitted acute viral hepatitis (Figure 1) and prevalent where sanitation is poor, it was first visualized in 1973 by immune electron microscopy in the feces of human volunteers [1]. Only a single HAV serotype exists, and it has never been shown to establish longterm persistent infections. Interest in HAV peaked in the late 1980s, but then declined with introduction of successful formalin-inactivated vaccines and the discovery of hepatitis $\mathrm{C}$ virus (HCV). $\mathrm{HCV}$, also a positive-strand RNA virus (family Flaviviridae), has a striking capacity to establish persistence and strong

(C) 2015 Elsevier B.V. All rights reserved.

Corresponding author: Stanley M. Lemon (smlemon@med.unc.edu), 8.034 Burnett-Womack CB \#7292, University of North Carolina at Chapel Hill, Chapel Hill, NC 27599-7292 USA, Tel: 1-919-843-1848; Fax: 1-919-843-7240.

Publisher's Disclaimer: This is a PDF file of an unedited manuscript that has been accepted for publication. As a service to our customers we are providing this early version of the manuscript. The manuscript will undergo copyediting, typesetting, and review of the resulting proof before it is published in its final citable form. Please note that during the production process errors may be discovered which could affect the content, and all legal disclaimers that apply to the journal pertain. 
association with chronic hepatitis, progressive hepatic fibrosis and liver cancer - clinical outcomes never linked to HAV. However, recent years have marked a resurgence of interest in HAV, sparked in part by the desire to understand these different infection outcomes. Many potential explanations have been advanced [2], but the mechanisms underlying $\mathrm{HCV}$ persistence remain elusive. Contrasts in the host response to HAV and HCV that are now emerging may provide important clues to this mystery. Recent studies have brought to light several unexpected aspects of the innate and adaptive immune response to HAV, and revealed paradigm-breaking features of HAV structure and the HAV lifecycle. Here, we review these recent developments and outline the questions they pose for future investigations.

\section{Structure of the infectious HAV particle}

While the organization of the HAV genome resembles other picornaviruses (Figure 2), recent crystallographic studies show the capsid to be intermediate in structure between that of 'primitive' insect dicistroviruses and mammalian picornaviruses such as poliovirus [3]. Although it was recognized that the HAV structure must differ from other picornaviruses given its impressive physical stability and a distinct morphogenesis pathway [4,5], the degree of difference comes as a surprise and indicates that HAV diverged from other picornaviruses eons ago. The capsid is also generally devoid of the surface topology that provides binding sites for cellular receptors on other picornaviruses [3], raising questions as to how HAV enters cells.

Even more surprising is the discovery that HAV is released from infected hepatocytes cloaked in host membranes and thereby hidden from neutralizing antibodies [6]. These membrane-wrapped virions ('eHAV') (Figure 2) are infectious and possess key attributes of conventional enveloped viruses, including loss of infectivity upon extraction with organic solvents. The membrane cloaking the virus is not decorated with virally-encoded glycoproteins, however, providing an important distinction and leading us to consider these eHAV virions to be "quasi-enveloped" [7]. While the largest of the 4 capsid proteins, VP1, is 274 amino acid residues in length in 'naked', non-enveloped HAV virions, it has an $8 \mathrm{kDa}$ carboxyterminal extension (pX, also known as $2 \mathrm{~A}$ ) and is approximately 71 residues longer in eHAV (VP1-pX) [6]. pX is unrelated to any other known protein. It plays a critical role in capsid assembly and likely eHAV envelopment, but is cleaved from the capsid upon loss of the membrane $[5,6]$.

The biogenesis of quasi-enveloped eHAV particles is dependent upon ALIX and VPS4B [6], components of the cellular endosomal sorting complex required for transport (ESCRT) commonly involved in budding of conventional enveloped viruses [8]. ALIX appears to bind tandem $\mathrm{YPX}_{3} \mathrm{~L}$ 'late domains' in VP2 [6]. Although confirmatory ultrastructural data are lacking, this likely promotes the budding of assembled capsids into multivesicular bodies (MVBs), leading to eHAV envelopment and a release mechanism resembling exosome biogenesis [9]. Since the VP2 late domains are buried beneath the surface of the naked capsid in the X-ray structure [3], the capsid appears to undergo significant conformational rearrangement upon membrane dissolution and loss of $\mathrm{pX}$. 
Only quasi-enveloped eHAV is detected in serum and plasma during acute infection, whereas non-enveloped virions are shed in feces [6]. While not well understood, these naked virions are probably produced in the liver and secreted in bile. They may be released from hepatocytes as eHAV, but converted to naked virions in the proximal biliary canaliculus where local bile salt concentrations could be sufficient to dissolve the membrane [10]. However, available data do not exclude an intestinal source [11]. The non-enveloped virion is remarkably stable to heat, low $\mathrm{pH}$, and drying, facilitating viral transmission $[3,4]$. This dual lifestyle, quasi-enveloped and cloaked from neutralizing antibodies within the host while devoid of membranes and stable in the environment, provides unique opportunities for spread within and between hosts.

Cellular entry of eHAV occurs via a chloroquine-sensitive endocytic pathway distinct from entry of non-enveloped virions [6]. Entry of both virion types is dependent upon the

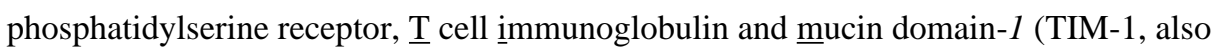
referred to as HAVCR1), but little else is known about this. Important questions that remain unresolved is how the eHAV membrane alters cellular tropism, and whether a distinct receptor is involved in eHAV entry.

\section{Innate and cell-intrinsic immune responses}

Type 1 interferon (IFN- $\alpha / \beta)$ is both a first line of defense against viruses and important in optimal priming of subsequent adaptive cellular immunity. HAV evokes a minimal intrahepatic type I IFN response in chimpanzees, far less quantitatively than that observed in acute HCV infections (Figure 3) [12]. Despite this, intrahepatic viral RNA is 100- to 1000fold more abundant in acute HAV versus HCV infection. There are several possible explanations for these differences. Both viruses express proteases that cleave MAVS and TRIF, key adaptor proteins in RIG-I-like receptor (RLR) and Toll-like receptor 3 (TLR3) signaling, respectively. This represents an interesting example of convergent evolution, as the responsible $\mathrm{HAV}$ proteases, $3 \mathrm{ABC}$ and $3 \mathrm{CD}[13,14]$, are structurally and phylogenetically unrelated to the HCV protease, NS3/4A [15]. However, the mature HAV protease, $3 \mathrm{C}^{\mathrm{pro}}$, also cleaves NEMO, a bridging adaptor required for NF- $\kappa \mathrm{B}$ activation and IFN- $\beta$ expression [16]. The targeting of NEMO by HAV may provide an additional level of disruption in interferon signaling beyond that imposed by $\mathrm{HCV}$, possibly contributing to less interferon-stimulated gene (ISG) expression in hepatitis A.

Differences may also exist in the plasmacytoid dendritic cell (pDC) response to these infections. pDCs are activated and produce IFN through a TLR7 pathway when placed in co-culture with HCV-infected cells [17]. Although they do not sense some picornaviruses unless the virus is complexed with antibodies [18,19], they do produce substantial amounts of IFN- $a$ when co-cultured with HAV-infected cells [20]. pDCs preferentially take up quasienveloped eHAV virions, which stimulate IFN production in the absence of genome replication. pDCs sense HCV RNA carried as cargo from infected cells by exosomes [21], a mechanistically similar process since eHAV resemble exosomes and may share a similar biogenesis. A key difference between HAV and HCV, however, may be in how pDCs are recruited to the liver. In chimpanzees, numerous pDCs are present within the liver by the end of the first week of HAV infection (Figure 1) [20]. For unknown reasons, they disappear 
and cannot be detected at the peak of virus replication and acute inflammation 2-3 weeks later. Less is known about temporal aspects of the pDC response in $\mathrm{HCV}$ infection, but pDCs appear to be abundant in chronically infected livers where ISG expression is often strong [22].

$\mathrm{HCV}$ may also replicate less efficiently than HAV, resulting in lower expression of $\mathrm{HCV}$ proteins and therefore less efficient antagonism of IFN signaling. HCV is exquisitely and uniquely sensitive to oxidative membrane damage, whereas HAV is not [23]. Because HCV infection induces oxidative stress, an auto-regulatory circuit unique to $\mathrm{HCV}$ may ensure that replication is maintained at low levels within the liver.

\section{Adaptive Immunity and Control of HAV Infection.}

HAV-specific humoral and cellular immune responses typically appear 4-5 weeks after infection with the onset of hepatitis (Figure 1). Increased numbers of plasmablasts secreting IgM with a variety of specificities are present at this point in time [24], but this rapidly transitions to a neutralizing IgG response that provides life-long protection from hepatitis A [25]. Passive transfer of anti-HAV antibodies or vaccination up to two weeks after exposure to the virus can prevent liver disease [26], indicating that antibodies also have the potential to modulate the course of an established infection. Neutralizing antibodies recognize a small number of closely-positioned epitopes in the highly conserved VP1, VP3 [27] and possibly VP2 [3] capsid proteins.

Non-enveloped HAV are readily neutralized when pre-treated with antibodies before inoculation onto cultured cells [28], and thus it has been assumed that immunization or immune globulins protect against disease by neutralizing circulating virus. However, quasienveloped eHAV virions (the only virion type found in blood) are completely resistant to neutralization in classical infectious focus-reduction assays since the membrane effectively cloaks the capsid [6]. Despite this, replication is inhibited when anti-capsid antibodies are added to cells several hours after eHAV infection [6]. Neutralization probably occurs within late endosomes or lysosomes, where the membrane is likely to be removed and the capsid exposed during entry of the virus. The kinetics of such post-endocytic neutralization suggest that eHAV entry is relatively slow, requiring 4-6 hours for dissolution of the membrane. In contrast, antibodies have no effect when added even immediately after infection of cells with non-enveloped HAV [6].

HAV-specific cytotoxic CD8+ T cell responses were first described in the blood [29] and liver [30] of jaundiced patients with acute hepatitis A 25 years ago. Since the icteric phase of infection typically coincides with a sharp decline in viremia, this CD8+ T cell response was correlated kinetically with control of virus replication (Figure 1). The detection of cytotoxic CD8+ T cells during this acute phase of the infection was also consistent with liver injury being immune mediated, since robust virus replication occurs during the preceding 2-3 week prodromal period without liver disease. However, a series of recent studies have provided fresh insight into cellular immune responses using newer methods for more precise measurement of T cell frequency and function. Epitopes presented by defined class I epitopes were mapped in human subjects infected during a recent hepatitis A outbreak [31]. 
Whereas CD8+ T cells targeting these epitopes were successfully expanded from blood of patients with acute HAV infection, most circulating CD8+ T cells were present at frequencies too low for direct visualization with class I tetramers. Effector functions were not assessed. A survey of CD4+ helper and CD8+ cytotoxic T cell activity was also undertaken in two chimpanzees with relatively mild transient hepatitis 3-4 weeks after experimental challenge [32]. In that study, CD8+ T cells were visualized in blood with class I tetramers, but they targeted few epitopes and did not gain effector functions until after viremia and hepatitis had substantially declined (Figure 1). On the other hand, multifunctional HAV-specific CD4+ T cells targeting over 30 discrete class II epitopes appeared in blood at much higher frequency well before CD8+ T cells were detected. Control of viremia was more closely linked to expansion of functional CD4+ T cells than CD8+ T cells [32]. The frequency of HAV-specific CD4+ T cells declined very slowly in blood after termination of viremia and fecal shedding of virus. Slow contraction of CD4+ T cells paralleled gradual clearance of HAV RNA from the liver over 8-9 months [12,32]. While the continued presence of intrahepatic HAV RNA for such a long period of time was unexpected, it is consistent with a role for residual viral antigen in prolonging CD4+ but not CD8+ T cell contraction as described recently in lymphocytic choriomeningitis virus (LCMV)-infected mice [33].

While the detection of CD8+ T cells in patients with acute hepatitis A provided an early conceptual framework explaining both acute liver injury and immune control of HAV (Figure 4, left panel), liver injury can range from inapparent to severe (even fatal) in acute HAV infection. This suggests that cytotoxic CD8+ T cell activity is likely to be a variable feature in hepatitis A. More recent studies of patients infected during a recent nationwide epidemic of hepatitis A in South Korea established an inverse correlation between the frequency and function of regulatory $\mathrm{T}$ cells (Treg) in blood and the severity of liver injury as reflected by increases in in serum alanine aminotransferase (ALT) [34]. The frequency of circulating HAV-specific CD8+ T cells did not correlate with Treg activity [34], raising questions about the identity of effector cells that mediate immunopathology. Little is known about the role of NK and NKT cells in this infection. Moreover, the potential for HAV to modulate NKT cell cytotoxic activity [35] as well as Treg function [36] through direct interaction of the viral capsid with the TIM-1 receptor highlights the complexity of host virus-interactions in this infection.

\section{Conclusion: Towards a more flexible model of HAV immunity and pathogenesis}

The new findings summarized above demand a rethinking of the relationships between innate and adaptive immune responses and acute liver injury, as well as immune elimination of HAV. A new model might place less emphasis on cytotoxic elimination of HAV-infected hepatocytes by CD8+ T cells, particularly if the delay in acquisition of effector function observed in chimpanzees [32] is recapitulated in future studies of human subjects (Figure 4, right panel). A defect in early antiviral effector function by CD8+ T cells is probably not caused by an absence of help because CD4+ T cells in the HAV-infected chimpanzees produced IL-2, IL-21, and IFN- $\gamma$ [32]. Interference with class I antigen processing and 
presentation has been described for other picornaviruses [37], but the relevance of this to generation of $\mathrm{CD} 8+\mathrm{T}$ cell immunity in HAV infection remains to be determined. Weak type I IFN responses in the liver [12] might also dampen CD8+ T cell immunity as this cytokine delivers an important differentiation signal in some virus infections. As discussed above, more needs to be learned about the fate of pDCs in the acutely infected liver in order to better understand the paucity of type I IFN responses and how it might limit development of CD8+ $\mathrm{T}$ cell responses.

The concept that $\mathrm{CD} 4+\mathrm{T}$ cells provide direct control of virus infections through production of antiviral cytokines is gaining favor [38]. Non-cytotoxic control of virus replication by $\mathrm{CD} 4+\mathrm{T}$ cells could be a general mechanism for terminating HAV infection regardless of CD8+ T cell activity. CD4+ T cells could also have a protective role in the postconvalescent phase of infection, when HAV RNA genomes are gradually lost from liver. Clinical relapse associated with apparent recrudescent infection after the initial resolution of symptoms of hepatitis A [39], and prolonged presence of HAV RNA in serum of some adults [40], suggests the existence of a non-cytotoxic mechanism of immune surveillance that is effective in most infections. CD4+ T cells, that contract gradually after apparent resolution of infection [32], could serve this function. However, the persistence of viral RNA in the liver for months after the cessation of fecal shedding [12] remains to be explained. One interesting possibility is that virions may remain complexed to neutralizing antibodies within endolysosomes.

Finally, despite its major fecal-oral route of transmission, the role of the gut as a site for HAV replication and perhaps as a regulator of immune responses to the virus has received insufficient attention [41]. Early studies in owl monkeys suggest HAV may replicate in the lower gastrointestinal tract [11], but this has never been confirmed in humans. Local inflammatory signals elicited by even limited replication of HAV in the gut could substantially influence the nature of immunity and disease severity in liver [41].

A better understanding of HAV pathogenesis and immunity could provide general insight into mechanisms of immune evasion and control of other viruses that infect the liver, including HCV. A focus on human subjects who are infected as a result of sporadic and epidemic spread of the virus provides one path forward. However, there is a need for a renewed effort to better characterize non-human primate models of hepatitis A and perhaps even the adaptation of HAV to replication in rodents to facilitate access to tissue and experimental manipulation of immune responses.

\section{Acknowledgements}

The authors gratefully acknowledge many stimulating conversations with Drs. Robert Lanford and Jason Whitmire concerning HAV pathogenesis, and wish to acknowledge Dr. Lanford's essential and invaluable contributions to the chimpanzee studies we review here. This work was supported in part by grants from the National Institutes of Health, R01-AI103083 and U19-AI109965 (SML). 


\section{REFERENCES}

1. Feinstone SM, Kapikian AZ, Purcell RH. Hepatitis A: detection by immune electron microscopy of a viruslike antigen associated with acute illness. Science. 1973; 182:1026-1028. [PubMed: 4356028]

2. Thimme R, Binder M, Bartenschlager R. Failure of innate and adaptive immune responses in controlling hepatitis C virus infection. FEMS Microbiol Rev. 2012; 36:663-683. [PubMed: 22142141]

3. Wang X, Ren J, Gao Q, Hu Z, Sun Y, Li X, Rowlands DJ, Yin W, Wang J, Stuart DI, et al. Hepatitis A virus and the origins of picornaviruses. Nature. 2014 [Epub ahead of print: doi: 10.1038/ nature13806]. The high resolution X-ray structure of both empty and full non-enveloped HAV particles reveals HAV to be a primitive picornavirus, with structural similarities to insect dicistroviruses. **

4. Siegl G, Weitz M, Kronauer G. Stability of hepatitis A virus. Intervirology. 1984; 22:218-226. [PubMed: 6096294]

5. Probst C, Jecht M, Gauss-Muller V. Intrinsic signals for the assembly of hepatitis A virus particles. Role of structural proteins VP4 and 2A. J Biol Chem. 1999; 274:4527-4531. [PubMed: 9988685]

6. Feng Z, Hensley L, McKnight KL, Hu F, Madden V, Ping L, Jeong S-H, Walker C, Lanford RE, Lemon SM. A pathogenic picornavirus acquires an envelope by hijacking cellular membranes. Nature. 2013; 496:367-371. [PubMed: 23542590] This study demonstrated that eHAV is released from infected hepatocytes cloaked in membranes via an ESCRT-dependent process, thereby providing a novel means of cellular egress for a "non-enveloped" virus. Only eHAV was detectable in serum or plasma from infected humans and chimpanzees. $* *$

7. Feng Z, Hirai-Yuki A, McKnight KL, Lemon SM. Naked viruses that aren't always naked: Quasienveloped agents of acute hepatitis. Ann Rev Virology. 2014; 1:539-560.

8. Votteler J, Sundquist WI. Virus budding and the ESCRT pathway. Cell Host Microbe. 2013; 14:232-241. [PubMed: 24034610]

9. Colombo M, Raposo G, Thery C. Biogenesis, secretion, and intercellular interactions of exosomes and other extracellular vesicles. Annu Rev Cell Dev Biol. 2014; 30:255-289. [PubMed: 25288114]

10. Boyer JL. Bile formation and secretion. Compr Physiol. 2013; 3:1035-1078. [PubMed: 23897680]

11. Asher LVS, Binn LN, Mensing TL, Marchwicki RH, Vassell RA, Young GD. Pathogenesis of hepatitis A in orally inoculated owl monkeys (Aotus trivergatus). J Med Virol. 1995; 47:260-268. [PubMed: 8551278]

12. Lanford RE, Feng Z, Chavez D, Guerra B, Brasky KM, Zhou Y, Yamane D, Perelson AS, Walker $\mathrm{CM}$, Lemon SM. Acute hepatitis A virus infection is associated with a limited type I interferon response and persistence of intrahepatic viral RNA. Proc Nat'l Acad Sci USA. 2011; 108:1122311228. [PubMed: 21690403]

13. Yang Y, Liang Y, Qu L, Chen Z, Yi M, Li K, Lemon SM. Disruption of innate immunity due to mitochondrial targeting of a picornaviral protease precursor. Proc Nat'l Acad Sci USA. 2007; 104:7253-7258. [PubMed: 17438296]

14. Qu L, Feng Z, Yamane D, Liang Y, Lanford RE, Li K, Lemon SM. Disruption of TLR3 signaling due to cleavage of TRIF by the hepatitis A virus protease-polymerase processing intermediate, 3CD. PLoS Pathog. 2011; 7(9):e1002169. [PubMed: 21931545]

15. Li XD, Sun L, Seth RB, Pineda G, Chen ZJ. Hepatitis C virus protease NS3/4A cleaves mitochondrial antiviral signaling protein off the mitochondria to evade innate immunity. Proc. Nat'1. Acad. Sci USA. 2005; 102:17717-17722. [PubMed: 16301520]

16. Wang D, Fang L, Wei D, Zhang H, Luo R, Chen H, Li K, Xiao S. Hepatitis A virus 3C protease cleaves NEMO to impair induction of beta interferon. J Virol. 2014; 88:10252-10258. [PubMed: 24920812] This report shows that the mature HAV protease, $3 \mathrm{C}^{\text {pro }}$, cleaves an adaptor protein that is essential for activation of NF- $\kappa \mathrm{B}$. It adds to earlier reports of cleavage of MAVS and TRIF by $3 \mathrm{C}$ protease precursors, expanding the scope of viral disruption of signaling pathways required for induction of type I IFN expression. * 
17. Takahashi K, Asabe S, Wieland S, Garaigorta U, Gastaminza P, Isogawa M, Chisari FV. Plasmacytoid dendritic cells sense hepatitis $C$ virus-infected cells, produce interferon, and inhibit infection. Proc Nat'l Acad Sci USA. 2010; 107:7431-7436. [PubMed: 20231459]

18. Guzylack-Piriou L, Bergamin F, Gerber M, McCullough KC, Summerfield A. Plasmacytoid dendritic cell activation by foot-and-mouth disease virus requires immune complexes. Eur J Immunol. 2006; 36:1674-1683. [PubMed: 16783856]

19. Wang JP, Asher DR, Chan M, Kurt-Jones EA, Finberg RW. Cutting Edge: Antibodymediated TLR7-dependent recognition of viral RNA. J Immunol. 2007; 178:3363-3367. [PubMed: 17339429]

20. Feng Z, Li Y, McKnight KL, Hensley L, Lanford RE, Walker CM, Lemon SM. Human pDCs preferentially sense enveloped hepatitis A virions. J Clin Invest. 2014 [Epub in advance of print: doi: 10.1172/JCI77527]. This report shows that high concentrations of quasi-enveloped eHAV virions stimulate plasmacytoid dendritic cells (pDCs) to secrete IFN-a, while also showing that pDCs are recruited to the chimpanzee liver only during the first week of infection. *

21. Dreux M, Garaigorta U, Boyd B, Decembre E, Chung J, Whitten-Bauer C, Wieland S, Chisari FV. Short-range exosomal transfer of viral RNA from infected cells to plasmacytoid dendritic cells triggers innate immunity. Cell Host Microbe. 2012; 12:558-570. [PubMed: 23084922]

22. Lau DT, Negash A, Chen J, Crochet N, Sinha M, Zhang Y, Guedj J, Holder S, Saito T, Lemon SM, et al. Innate immune tolerance and the role of kupffer cells in differential responses to interferon therapy among patients with HCV genotype 1 infection. Gastroenterology. 2013; 144:402-413. [PubMed: 23123437]

23. Yamane D, McGivern DR, Wauthier E, Yi M, Madden VJ. Regulation of the hepatitis C virus RNA replicase by endogenous lipid peroxidation. Nat Med. 2014; 20:927-935. [PubMed: 25064127]

24. Hong S, Lee HW, Chang DY, You S, Kim J, Park JY, Ahn SH, Yong D, Han KH, Yoo OJ, et al. Antibody-secreting cells with a phenotype of Ki-67low, CD138high, CD31high, and CD38high secrete nonspecific IgM during primary hepatitis A virus infection. J Immunol. 2013; 191:127134. [PubMed: 23729443]

25. Lemon SM, Binn LN. Serum neutralizing antibody response to hepatitis A virus. J Infect Dis. 1983; 148:1033-1039. [PubMed: 6317766]

26. Victor JC, Monto AS, Surdina TY, Suleimenova SZ, Vaughan G, Nainan OV, Favorov MO, Margolis HS, Bell BP. Hepatitis A vaccine versus immune globulin for postexposure prophylaxis. N Engl J Med. 2007; 357:1685-1694. [PubMed: 17947390]

27. Ping LH, Lemon SM. Antigenic structure of human hepatitis A virus defined by analysis of escape mutants selected against murine monoclonal antibodies. J Virol. 1992; 66:2208-2216. [PubMed: 1312628]

28. Lemon SM, Murphy PC, Provost PJ, Chalikonda I, Davide JP, Schofield TL, Nalin DR, Lewis JA. Immunoprecipitation and virus neutralization assays demonstrate qualitative differences between protective antibody responses to inactivated hepatitis A vaccine and passive immunization with immune globulin. J Infect Dis. 1997; 176:9-19. [PubMed: 9207344]

29. Maier K, Gabriel P, Koscielniak E, Stierhof YD, Wiedmann KH, Flehmig B, Vallbracht A. Human gamma interferon production by cytotoxic T lymphocytes sensitized during hepatitis A virus infection. J Virol. 1988; 62:3756-3763. [PubMed: 2843673]

30. Fleischer B, Fleischer S, Maier K, Wiedmann KH, Sacher M, Thaler H, Vallbracht A. Clonal analysis of infiltrating T lymphocytes in liver tissue in viral hepatitis A. Immunology. 1990; 69:14-19. [PubMed: 2312151]

31. Schulte I, Hitziger T, Giugliano S, Timm J, Gold H, Heinemann FM, Khudyakov Y, Strasser M, Konig C, Castermans E, et al. Characterization of CD8+ T-cell response in acute and resolved hepatitis A virus infection. J Hepatol. 2010

32. Zhou Y, Callendret B, Xu D, Brasky KM, Feng Z, Hensley LL, Guedj J, Perelson AS, Lemon SM, Lanford RE, et al. Dominance of the CD4+ T helper cell response during acute resolving hepatitis A virus infection. J Exp Med. 2012; 209:1481-1492. [PubMed: 22753925] This study provided the first analysis of CD4+ T helper cell immunity against HAV, showing that this response is earlier, broader, and more functional than the CD8+ T cell response in chimpanzees. It suggested 
the potential for non-cytotoxic control of HAV infection and perhaps immune surveillance in liver where HAV genomes decay slowly over several months following resolution of viremia. **

33. Misumi I, Alirezaei M, Eam B, Su MA, Whitton JL, Whitmire JK. Differential T cell responses to residual viral antigen prolong CD4+ T cell contraction following the resolution of infection. $\mathrm{J}$ Immunol. 2013; 191:5655-5668. [PubMed: 24146043]

34. Choi YS, Lee J, Lee HW, Chang DY, Sung PS, Jung MK, Park JY, Kim JK, Lee JI, Park H, et al. Liver injury in acute hepatitis $\mathrm{A}$ is associated with decreased frequency of regulatory $\mathrm{T}$ cells caused by Fas-mediated apoptosis. Gut. 2014 [Epub ahead of print, doi: 10.1136/ gutjnl-2013-306213]. This study demonstrated a decrease in Treg frequency during acute hepatitis A that was inversely associated with severity of liver disease but not HAV-specific CD8+ T cell activity. *

35. Kim HY, Eyheramonho MB, Pichavant M, Gonzalez Cambaceres C, Matangkasombut P, Cervio G, Kuperman S, Moreiro R, Konduru K, Manangeeswaran M, et al. A polymorphism in TIM1 is associated with susceptibility to severe hepatitis A virus infection in humans. J Clin Invest. 2011; 121:1111-1118. [PubMed: 21339644]

36. Manangeeswaran M, Jacques J, Tami C, Konduru K, Amharref N, Perrella O, Casasnovas JM, Umetsu DT, Dekruyff RH, Freeman GJ, et al. Binding of hepatitis A virus to its cellular receptor 1 inhibits T-regulatory cell functions in humans. Gastroenterology. 2012; 142:1516-1525. e1513. [PubMed: 22430395]

37. Kemball CC, Harkins S, Whitmire JK, Flynn CT, Feuer R, Whitton JL. Coxsackievirus B3 inhibits antigen presentation in vivo, exerting a profound and selective effect on the MHC class I pathway. PLoS Pathog. 2009; 5:e1000618. [PubMed: 19834548]

38. Sant AJ, McMichael A. Revealing the role of CD4(+) T cells in viral immunity. J Exp Med. 2012; 209:1391-1395. [PubMed: 22851641]

39. Sjogren MH, Tanno H, Fay O, Sileoni S, Cohen BD, Burke DS, Feighny RJ. Hepatitis A virus in stool during clinical relapse. Ann Intern Med. 1987; 106:221-226. [PubMed: 3026213]

40. Normann A, Jung C, Vallbracht A, Flehmig B. Time course of hepatitis A viremia and viral load in the blood of human hepatitis A patients. J Med Virol. 2004; 72:10-16. [PubMed: 14635005]

41. Adams DH, Eksteen B, Curbishley SM. Immunology of the gut and liver: a love/hate relationship. Gut. 2008; 57:838-848. [PubMed: 18203807] 


\section{HIGHLIGHTS}

- Circulating virions are cloaked in membranes and resistant to neutralization

- Acute HAV infection induces a meager intrahepatic type I interferon response

- CD4+T cells appear earlier and acquire effector function before CD8+ T cells

- Immune control of HAV may be predominantly non-cytotoxic and cytokine driven 

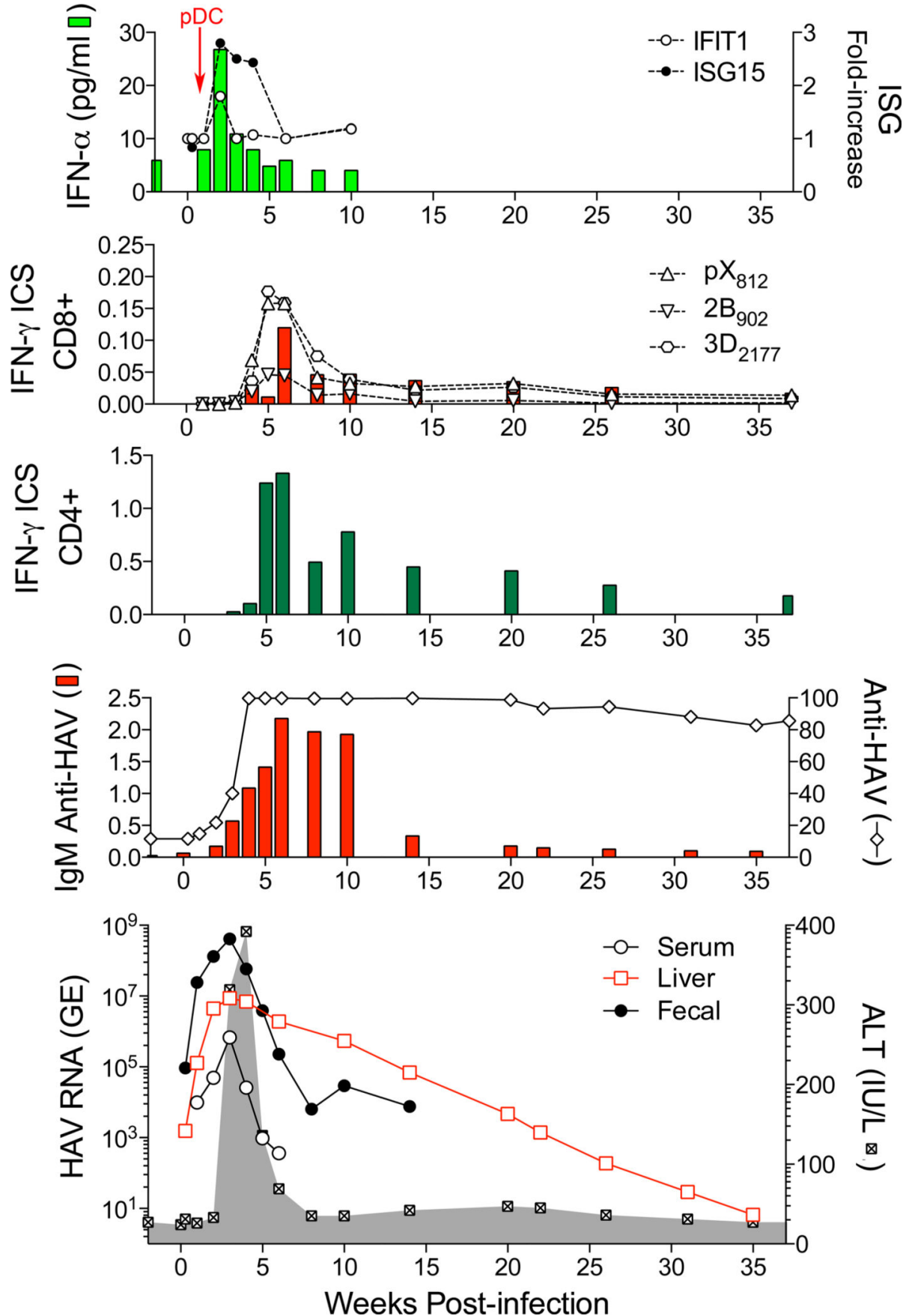

Figure 1.

Virologic and immunologic events during acute HAV infection in a chimpanzee inoculated intravenously with wild-type HAV [12,20,32]. Working from the bottom up, the lowest panel shows the presence of viral RNA (GE, genome equivalents) in serum $(\mathrm{GE} / \mathrm{ml})$, feces (GE/gm), and liver tissue (GE/ $\mu \mathrm{g}$ total RNA) in relationship to serum alanine aminotransferase (ALT) activity shown in the shaded zone [12]. The prolonged persistence of intrahepatic HAV RNA is surprising. The panel immediately above shows total anti-HAV antibody (\% blocking in a competitive ELISA assay) and IgM anti-HAV (ELISA O.D.) 
[12]. The next two panels show frequencies of HAV-specific CD4+ and CD8+ T cells among peripheral blood mononuclear cells, as determined in an IFN $-\gamma$ intracellular staining (ICS) assay [32]. CD8+ cells were also quantified on the basis of staining with tetramers targeting epitopes in $\mathrm{pX}, 2 \mathrm{~B}$, and $3 \mathrm{D}^{\mathrm{pol}}$ (see Figure 2). Note the difference in scale between CD4+ and CD8+ T cell frequencies. The top panel shows type I IFN responses to HAV infection as reflected in minimal and only early serum IFN-a levels detectable by cytokine ELISA, and minimal increases in intrahepatic expression of IFN-stimulated genes: IFIT1 and ISG15 [12]. pDCs were detected in liver tissue only at 1 week after viral challenge (arrow) [20]. (The authors gratefully acknowledge the essential involvement of Dr. Robert Lanford in these comprehensive chimpanzee studies.) 

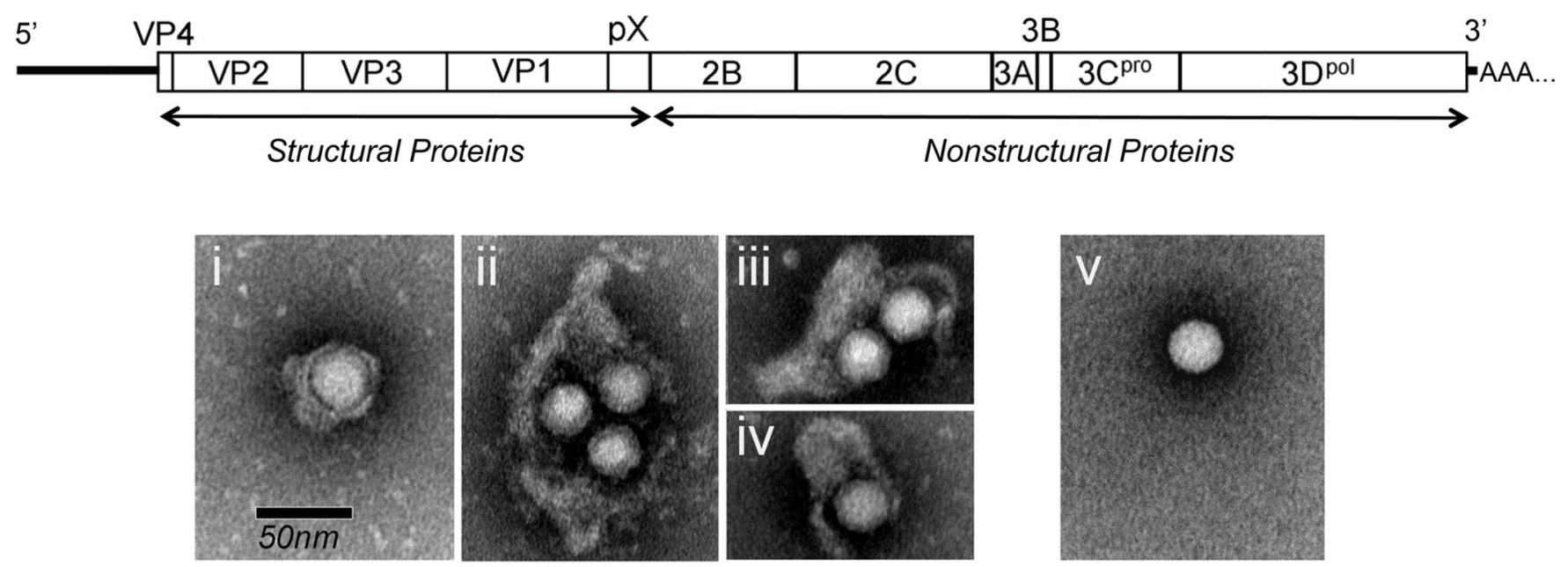

Figure 2.

Schematic showing organization of the $7.5 \mathrm{~kb}$ single-stranded, positive-sense RNA genome of HAV. The 2227 amino acid residue polyprotein is comprised of both structural and nonstructural proteins, and is flanked by 5 . and 3. untranslated RNA segments containing regulatory elements. Below are shown electron microscopic images of gradient purified quasi-enveloped eHAV (panels i-iv) and naked, non-enveloped HAV (panel v) released from infected hepatoma cell cultures. (Reproduced with permission from Feng et al. Nature 2013, 496:367-371). 


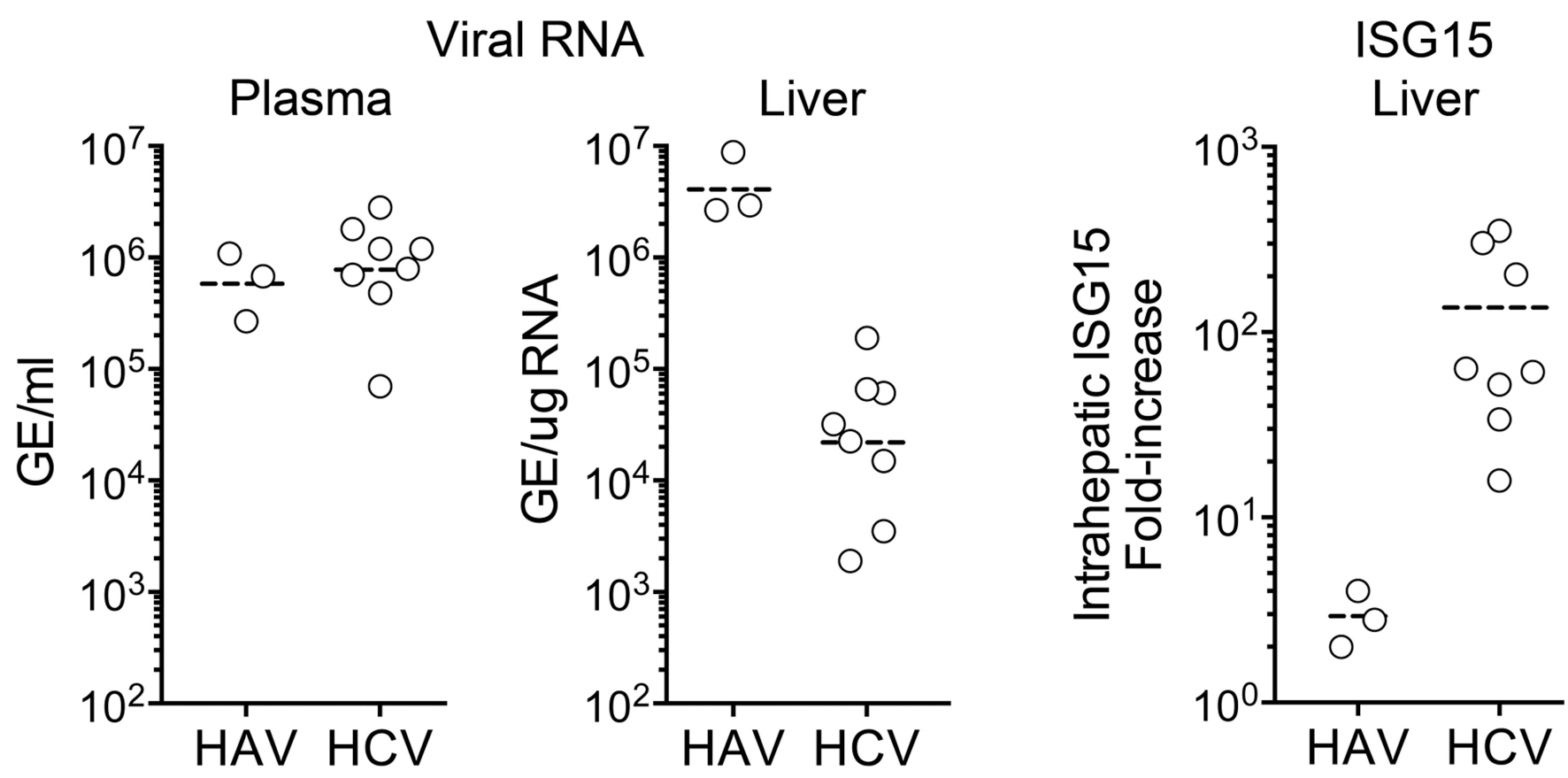

Figure 3.

Comparison of maximum intrahepatic and serum viral RNA abundance and interferonstimulated gene (ISG15) expression in acute, resolving HAV $(\mathrm{n}=3)$ and HCV $(\mathrm{n}=8)$ infections in experimentally infected chimpanzees. Differences in intrahepatic genome copy numbers $(p=0.01)$ and ISG expression $(p=0.01)$ were significant by two-sided MannWhitney test. Adapted from Lanford et al. [12]. 


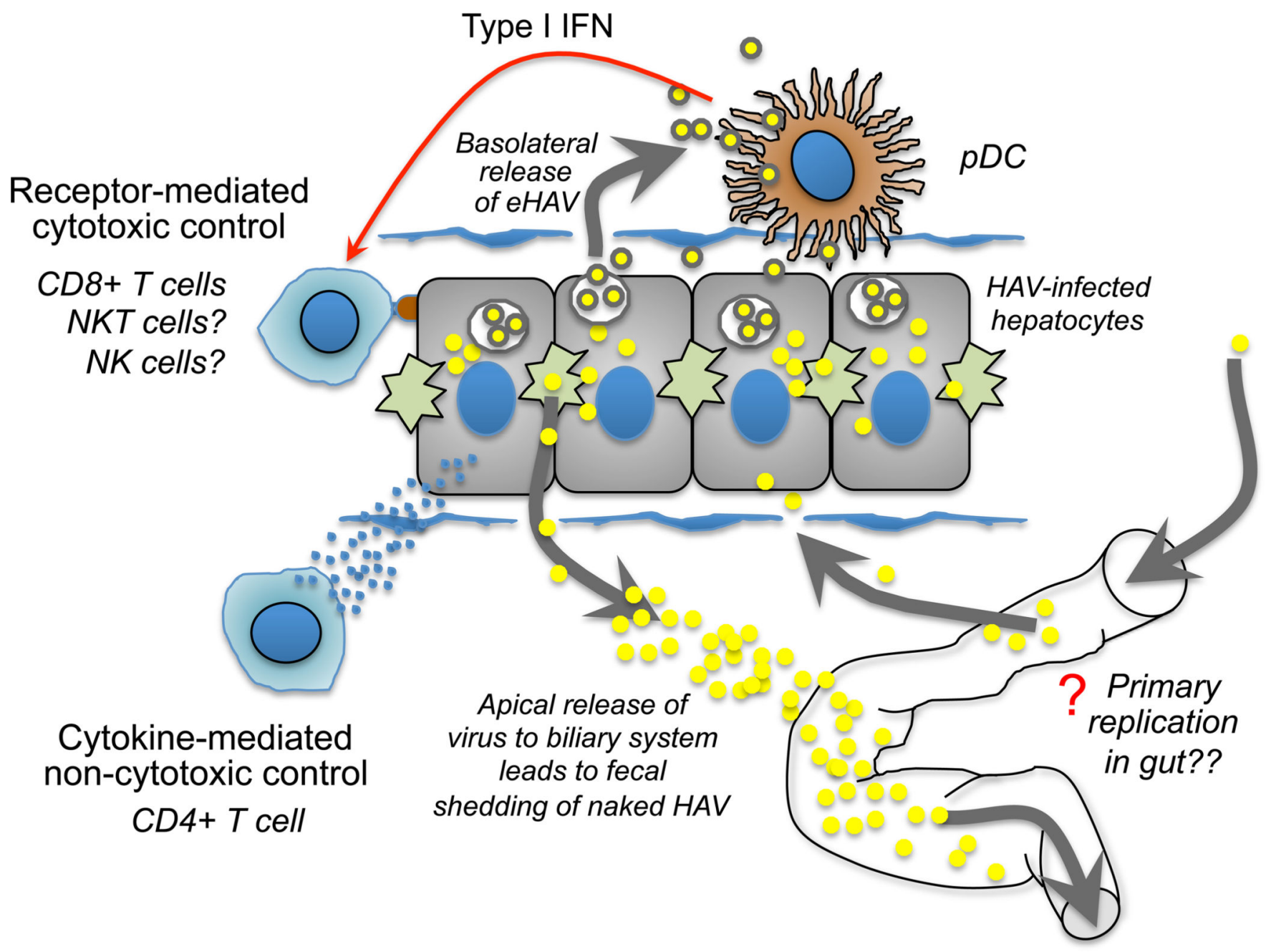

Figure 4.

Proposed cellular interactions in the liver during acute hepatitis A. HAV infection of the liver is thought to be non-cytopathic, resulting in release of quasi-enveloped virions from the basolateral plasma membrane of hepatocytes into the circulation and apical release of virus into the biliary system resulting in fecal shedding of naked HAV virions [6]. Contact between infected hepatocytes and plasmacytoid dendritic cells (pDC) results in transfer of eHAV to pDCs and signaling for production of type I IFN [20], a cytokine important to development of adaptive cellular immune responses. Cytotoxic cells, including virusspecific CD8+ T cells, NK cells, and NKT cells, have been implicated in acute hepatocellular injury during HAV infection. Liver damage can range from mild and inapparent, to severe and fatal, and may be regulated in part by the strength of the innate immune response, including type I IFN production by pDC. We propose that non-cytotoxic control of HAV replication is a central feature of infection and immune control of the virus regardless of disease severity. CD4+T helper cells are a potential source of antiviral cytokines because they appear in blood and acquire effector function earlier than CD8+ T cells [32], as shown in Figure 1. A primary site of virus replication within the gut after per- 
oral infection with the virus, or secondary to shedding of virus from the liver, remains speculative but could influence infection and immunity in the liver. 